\title{
Neural Basis of Dyslexia: A Comparison between Dyslexic and Nondyslexic Children Equated for Reading Ability
}

\author{
Fumiko Hoeft, ${ }^{1}$ Arvel Hernandez, ${ }^{1}$ Glenn McMillon, ${ }^{1}$ Heather Taylor-Hill, ${ }^{1}$ Jennifer L. Martindale, ${ }^{1}$ Ann Meyler, ${ }^{2}$ \\ Timothy A. Keller, ${ }^{2}$ Wai Ting Siok, ${ }^{1}$ Gayle K. Deutsch, ${ }^{1}$ Marcel Adam Just, ${ }^{2}$ Susan Whitfield-Gabrieli, ${ }^{1}$ and \\ John D. E. Gabrieli ${ }^{1}$ \\ ${ }^{1}$ Department of Psychology, Stanford University, Palo Alto, California 94305-2130, and ${ }^{2}$ Center for Cognitive Brain Imaging, Department of Psychology, \\ Carnegie Mellon University, Pittsburgh, Pennsylvania 15213-3890
}

\begin{abstract}
Adults and children with developmental dyslexia exhibit reduced parietotemporal activation in functional neuroimaging studies of phonological processing. These studies used age-matched and/or intelligence quotient-matched control groups whose reading ability and scanner task performance were often superior to that of the dyslexic group. It is unknown, therefore, whether differences in activation reflect simply poorer performance in the scanner, the underlying level of reading ability, or more specific neural correlates of dyslexia. To resolve this uncertainty, we conducted a functional magnetic resonance imaging study, with a rhyme judgment task, in which we compared dyslexic children with two control groups: age-matched children and reading-matched children (younger normal readers equated for reading ability or scanner-performance to the dyslexic children). Dyslexic children exhibited reduced activation relative to both age-matched and reading-matched children in the left parietotemporal cortex and five other regions, including the right parietotemporal cortex. The dyslexic children also exhibited reduced activation bilaterally in the parietotemporal cortex when compared with children equated for task performance during scanning. Nine of the 10 dyslexic children exhibited reduced left parietotemporal activation compared with their individually selected age-matched or reading-matched control children. Additionally, normal reading fifth graders showed more activation in the same bilateral parietotemporal regions than normal-reading third graders. These findings indicate that the activation differences seen in the dyslexic children cannot be accounted for by either current reading level or scanner task performance, but instead represent a distinct developmental atypicality in the neural systems that support learning to read.
\end{abstract}

Key words: dyslexia; age-matched; reading ability-matched; parietotemporal region; fMRI; phonological processing

\section{Introduction}

Dyslexia, which may affect 5-17\% of children (Shaywitz, 1998; Shaywitz and Shaywitz, 2003), is characterized by inaccurate and/or slow, effortful reading that typically originates with weakness in the phonological processing of language (Lundberg et al., 1980; MacDonald and Cornwall, 1995; Elbro et al., 1998; Shaywitz et al., 1999; Castles and Coltheart, 2004). Although there is substantial evidence of brain abnormalities in dyslexia (Shaywitz, 1998), the underlying cause of dyslexia remains unknown. Func-

Received Nov. 18, 2005; revised Aug. 24, 2006; accepted Aug. 29, 2006.

This work was supported by grants from the William and Flora Hewlett Foundation and the Richard King Mellon Foundation. Participants were recruited and characterized by the Power4Kids program, which is funded through a public-private partnership including the Haan Foundation for Children, Heinz Endowments, Grable Foundation, and the United States Department of Education. For a full description of this project, see http://www.haan4kids.org/ power4kids/. We thank Zsofia Huhn and Aaron Koralek for technical support.

Correspondence should be addressed to Fumiko Hoeft, 401 Quarry Road, Stanford, CA 94305-5795. E-mail: fumiko@stanford.edu.

F. Hoeft's present address: Center for Interdisciplinary Brain Sciences Research, Department of Psychiatry and Behavioral Sciences, Stanford University School of Medicine, Palo Alto, CA 94305-5795.

H. Taylor-Hill's present address: Department of Psychology, University of California, Los Angeles, CA 90095-1563.

W. T. Siok's present address: School of Humanities and State Key Laboratory of Brain and Cognitive Sciences, University of Hong Kong, Hong Kong, China.

S. Whitfield-Gabrieli and J. D. E Gabrieli's present address: Harvard-MIT Division of Health Sciences and Technology and Department of Brain and Cognitive Sciences, Massachusetts Institute of Technology, Cambridge, MA 02139. DOI:10.1523/JNEUROSCI.4931-05.2006

Copyright $\odot 2006$ Society for Neuroscience ～0270-6474/06/2610700-09\$15.00/0 tional neuroimaging studies examining phonological processing in dyslexic adults and children have consistently found reduced or absent activations in the left parietotemporal region, with the precise location varying across studies (for review, see Temple, 2002).

It is unknown, however, whether reduced activations in dyslexia reflect poor task performance in the scanner, reduced reading ability, or a dyslexia-specific deficit that is not simply accounted for by scanner task performance or reading ability. Previous imaging studies have compared activation between agematched and/or intelligence quotient (IQ)-matched control and dyslexic groups. This comparison introduces a confound between groups in their performance on phonological or other reading and language tasks. In all previous imaging studies, the dyslexic group had worse reading ability than the control group, and in most studies the dyslexic group performed 10-20\% worse on the scanner task (Temple et al., 2001; Shaywitz et al., 1998, 2002) (but see Brunswick et al., 1999; Eden et al., 2004). Therefore, the dyslexic group was often performing a more difficult task, and more task difficulty is associated with greater demands on attention, effort, and error-monitoring. In addition, typical developmental improvement in reading is associated with changes in brain activation in the absence of any reading deficit (Schlaggar et al., 2002; Shaywitz et al., 2002; Turkeltaub et al., 
Table 1. Demographics of the three groups

\begin{tabular}{|c|c|c|c|c|c|}
\hline Group & $\begin{array}{l}\text { 5th grade dyslexic } \\
{[\text { mean (SD)] }}\end{array}$ & $\begin{array}{l}\text { 5th grade age-matched } \\
{[\text { mean }(S D)]}\end{array}$ & $\begin{array}{l}\text { 3rd grade reading-matched } \\
{[\text { mean }(S D)]}\end{array}$ & $\begin{array}{l}\text { One-way ANOVA } \\
{\left[F_{(2,27)} ; p\right]}\end{array}$ & Post hoc $(p)$ \\
\hline Age & $11.37(0.73)$ & $10.95(0.33)$ & $8.75(0.30)$ & $81.68 ;<0.001$ & $0.16^{a} ;<0.001^{b} ;<0.001^{c}$ \\
\hline Gender & 4 female $/ 6$ male & 6 female $/ 4$ male & 5 female $/ 5$ male & & \\
\hline Word ID-raw & $51.4(5.87)$ & $69.0(6.22)$ & $51.6(6.04)$ & $27.93 ;<0.001$ & $<0.001^{a} ; 1.00^{b} ;<0.001^{c}$ \\
\hline Word ID-ss(age) & $78.9(4.12)$ & $96.7(5.03)$ & $96.6(4.93)$ & $47.31 ;<0.001$ & $<0.001^{a} ;<0.001^{b} ; 1.00^{c}$ \\
\hline Word attack-ss(age) & $84.1(7.46)$ & $96.9(6.44)$ & $96.0(3.94)$ & $13.59 ;<0.001$ & $<0.001^{a} ; 0.001^{b} ; 0.94^{c}$ \\
\hline Passage comprehension-ss(age) & $83.1(9.68)$ & $99.8(5.57)$ & $100.6(4.67)$ & $19.99 ;<0.001$ & $<0.001^{a} ;<0.001^{b} ; 0.97^{c}$ \\
\hline PPVT-ss(age) & $90.0(9.88)$ & $99.7(10.82)$ & $95.0(16.78)$ & $1.33 ; 0.29$ & \\
\hline Task accuracy & $70.5(17.2)$ & $90.0(10.5)$ & $80.0(12.5)$ & $5.06 ; 0.01$ & $0.01^{a} ; 0.28^{b} ; 0.25^{c}$ \\
\hline Correct reaction time (ms) & $2863(411)$ & $2445(428)$ & $2871(431)$ & $3.82 ; 0.04$ & $0.06^{a} ; 1.00^{b} ; 0.06^{c}$ \\
\hline Incorrect reaction time (ms) & $2805(704)$ & $2655(598)$ & $2950(528)$ & $0.40 ; 0.67$ & \\
\hline Absolute motion (mm) & $0.72(0.62)$ & $0.58(0.30)$ & $0.44(0.28)$ & $0.99 ; 0.39$ & \\
\hline Relative motion (mm) & $0.13(0.05)$ & $0.12(0.09)$ & $0.10(0.08)$ & $0.55 ; 0.58$ & \\
\hline
\end{tabular}

${ }^{a}$ Dyslexic versus age-matched.

${ }^{b}$ Dyslexic versus reading-matched.

'Age- versus reading-matched.

2003). Hence, activation differences between dyslexic and agematched groups could simply reflect a delay in the growth of a typical pattern of brain activation. These alternative interpretations can be addressed by including a second control group consisting of normal readers matched to the dyslexic group for current reading ability and by matching task performance in the scanner. Several behavioral studies have adopted this method (Bradley and Bryant, 1978, 1983; Stanovich et al., 1997; SprengerCharolles et al., 2000).

Here we report a functional magnetic resonance imaging (fMRI) study of phonological processing that disentangles the neural correlates of differences in reading performance from the distinct neural correlates of dyslexia. In this study, fifth-grade dyslexic children were compared with two different control groups, age-matched fifth graders and reading-matched third graders. We expected dyslexic children to show reduced activations relative to the age-matched control group. The critical and novel comparison was that with the reading-matched control group. If activation differences in dyslexia reflect reading level or scanner task performance, then areas of reduced activation in dyslexic children should have activation equal to that of third graders when equated for reading level or scanner task performance. If activation differences reflect specific neural correlates of dyslexia, then areas of reduced activation in dyslexic children should differ from that of reading-matched third graders.

\section{Materials and Methods}

Subjects. A total of 30 healthy, right-handed, native English speakers between $8-12$ years of age (mean, 10.4; SD, 1.3) participated in our study. All participants had no neurological or psychiatric disorders, were not on any medication, and had no contraindications to MRI. Reading ability was assessed for all subjects using a standard battery of behavioral measures. All subjects were recruited from a larger behavioral study of children in the Pittsburgh, PA, area. All protocols were approved by the University of Pittsburgh and Carnegie Mellon University Institutional Review Boards, and informed assent and consent was obtained for participation from each child and guardian, respectively.

Behavioral evaluation. Behavioral evaluations of reading and readingrelated skills were obtained by Mathematica Policy Research (Princeton, NY). Criteria for dyslexia were met if a child's age-adjusted [ss(age)] performance on the Woodcock Reading Mastery Tests (WRMT) word identification (ID) subtest fell below one SD from the norm [norm, 100; $1 \mathrm{SD}, 15$; i.e., ID-ss(age) < 85]. The ID test is a widely used measure of decoding skills often used as a criterion for defining dyslexia (Stanovich, 1999). Other neuroimaging studies of dyslexic children have used similar criteria (Simos et al., 2000a,b, 2002; Temple et al., 2001, 2003; Shaywitz et al., 2002, 2004; Aylward et al., 2003). All children were also tested on the Peabody Picture Vocabulary Test (PPVT), a test of receptive vocabulary. Significantly lower standardized reading scores (ID) than standardized receptive vocabulary scores (PPVT) define a discrepancy between impaired reading ability and relatively intact general language skills. Further, the PPVT is highly correlated with full scale IQ (0.90) in children on the Wechsler Intelligence Scale for Children (WISC III) (Dunn and Dunn, 1997), so lower reading than PPVT scores indicates a discrepancy between reading ability and IQ.

Subject groups. There were three groups: a dyslexic group and two control groups (Table 1). The dyslexic group consisted of 10 children (four female, six male) in grade 5 identified as dyslexic. The age-matched control group included 10 children (six female, four male) in grade 5, with decoding skills in the normal range [ID-ss(age) $>85$ ], who were matched to children in the dyslexic group by their chronological age. The reading-matched control group included 10 children (five female, five male) in grade 3, with decoding skills in the normal range [ID-ss(age) > 85], who were matched to children in the dyslexic group by their reading age. That is, each child in this group was matched by their absolute decoding ability, as measured by their raw ID score (ID-raw), to a child in the dyslexic group. Whereas the dyslexic group had low decoding skills relative to their age, the reading-matched group had age-appropriate decoding skills. A second matching procedure was derived from the ID standardized score [ID-ss(age)] which converts the raw (actual) score into an age-normalized score. The ID-ss(age) of each child in the agematched group was matched to that of a child in the reading-matched group. This ensured that reading ability relative to age was invariant between the two control groups.

Task design. A word-rhyme task was used in the scanner in which there were two conditions: rhyme and rest. During the rhyme condition, participants judged whether or not two visually presented words rhymed (e.g., bait, gate) or not (e.g., price, miss), and indicated each response with a right- or left-handed button press, respectively. Word pairs were selected so that the visual appearance of the last letters of the two words could not be used to determine whether they rhymed. Stimuli were balanced for frequency of occurrence, number of letters, and syllables between the rhyme and nonrhyme trials and across blocks (Zeno et al., 1995) (for the full list of stimuli, see supplemental Table 1, available at www.jneurosci.org as supplemental material). Each trial lasted a total of $6 \mathrm{~s}$, consisting of a $4 \mathrm{~s}$ period where the two words were presented simultaneously followed by a $2 \mathrm{~s}$ fixation cross. Each task block consisted of a $2 \mathrm{~s}$ cue period followed by five trials ( $32 \mathrm{~s}$ total). During the rest block, subjects saw a $15 \mathrm{~s}$ fixation cross on the screen. The entire scan was $234 \mathrm{~s}$ long, including two practice trials at the beginning, and consisted of four rhyme blocks and five rest blocks. Measures of task accuracy and reaction time (RT) were obtained.

Image acquisition. The MRI imaging and imaging-related procedures were performed at the Brain Imaging Research Center (Carnegie-Mellon 
University, University of Pittsburgh, Pittsburgh, PA). A 3.0 T Allegra scanner was used (Siemens, Malvern, PA). A T2*-weighted gradient echo, resonant echo planar pulse sequence sensitive to blood oxygen level-dependent contrast was used with the following acquisition parameters: repetition time, 1000 ; echo-time, 30 ; flip-angle, $60^{\circ}$; field-of-view, $20 \times 20 \mathrm{~cm}$; matrix-size, $64 \times 64$; axial-oblique plane with 16 slices, and a voxel size of $3.12 \times 3.12 \times 6 \mathrm{~mm}$ with a $1 \mathrm{~mm}$ gap. The number of slices did not provide consistent coverage of the sensorimotor cortex and cerebellum.

fMRI data analysis. Statistical analysis was performed using statistical parametric mapping software (SPM99; Wellcome Department of Cognitive Neurology, London, UK). After image reconstruction, each subject's data were slice-time corrected (ascending, reference slice 8), and realigned to the first functional volume. Sessions were then normalized using the mean functional volume resampled to $2 \times 2 \times 2 \mathrm{~mm}$ voxels in Montreal Neurological Institute (MNI) stereotaxic space (12 nonlinear iterations, $7 \times 8 \times 7$ nonlinear basis functions, medium regularization, sinc interpolation). Spatial smoothing was done with a Gaussian filter (8 $\mathrm{mm}$ full width at half maximum). Each voxel was scaled to the global signal of each volume, and each subject's data were high-pass filtered at $96 \mathrm{~s}$, and was analyzed using a fixed-effects model incorporating their six motion parameters $(x, y, z$, pitch, roll, yaw) as regressors to account for motion effects. Head motion was analyzed for absolute motion and reference motion (velocity) and showed no significant difference between groups (Jenkinson et al., 2002) (Table 1).

An adult template was used for normalization. There is evidence indicating minimal anatomical differences between children, ages 7 and 8, and adults relative to the resolution of fMRI data (Burgund et al., 2002), and minimal difference in functional foci between adults and children (Kang et al., 2003). Our study compared relatively older children of similar age-range across groups ( 8 to 12 years old); hence, the degree to which there were minor variations from the adult template would be similar across groups. Nevertheless, there could be differences in brain structures between dyslexic and nondyslexic subjects. We compared total gray matter, white matter, and CSF volumes between groups using oneway ANOVA of segmented high-resolution anatomical images. We found no significant effects of group ( $p=0.99$ for gray matter, $p=0.75$ for white matter, and $p=0.65$ for CSF). Therefore, activation differences cannot be accounted for by gross variations in gray matter volumes, but the influence of more subtle regional variation in anatomy on activation patterns cannot be determined.

Group analysis was performed with a random-effects model (Friston et al., 1999) using the rhyme versus rest contrast images (one per subject) including all subjects to show common regions of activation involved in rhyme judgment. Next, a two-sample $t$ test was conducted between the dyslexic and age-matched groups. We identified all regions at a significance level of $p<0.001$, uncorrected, and extent threshold (ET) of 10 voxels that showed significantly greater activation in the age-matched compared with the dyslexic groups (a total of six regions) (see Fig. 3), and greater activation in the dyslexic compared with the age-matched groups (no region survived this threshold). These regions were then defined as functional regions of interest (ROIs) in which to conduct additional analyses. This was done to reproduce previous findings and to identify regions that show differences in brain activation between dyslexic and age-matched groups. In addition, we performed a one-way ANOVA including all three groups (age-matched, reading-matched and dyslexic groups) as a complementary analysis to examine whether these regions were similar to what was found in the age-matched versus dyslexic group comparison (supplemental Fig. 1 and Table 2, available at www.jneurosci. org as supplemental material).

Contrast values were extracted from each subject in each of the six ROIs (effect size calculated as the linear combination of $\beta$ parameters). These values were compared among groups using a one-way ANOVA for each ROI; post hoc analyses were performed when there was a significant effect. We also extracted $\beta$ estimates of the rest and rhyme conditions in the same manner to investigate whether the differences in contrast values were arising from differences in the resting-baseline condition or the rhyme condition. To examine whether activation in the left parietotemporal region correlated with reading ability, simple correlation analyses
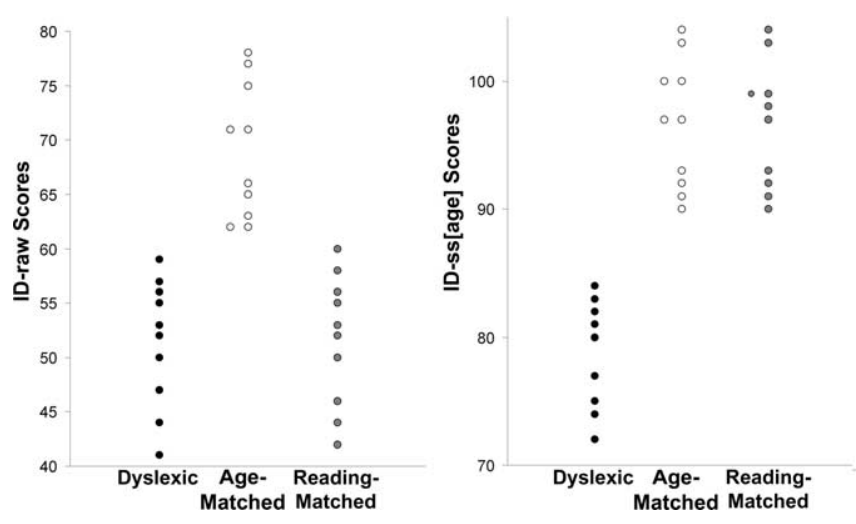

Figure 1. Scatter plots of ID scores for the three groups. ID raw scores (ID-raw) are on the left and ID standardized scores [ID-ss(age)] are on right.

were performed in the left parietotemporal region between the IDss(age), WRMT word attack subtest [WA-ss(age)], and WRMT passage comprehension subtest [PC-ss(age)] scores and contrast values. Finally, to examine the effect of differences in scanner task performance on brain activation, two-sample $t$ tests were computed between dyslexic and reading-matched groups only including subjects matched by scanner task performance.

Group contrast images were overlaid onto the SPM99 highresolution $\mathrm{T} 1$ individual template image and viewed in the sagittal, coronal, and axial views or a three-dimensional (3D) rendered brain. Coordinates of activation were converted from MNI to Talairach space using the mni2tal function (http://www.mrc-cbu.cam.ac.uk/ Imaging/Common/mnispace.shtml). Coordinates $x, y$, and $z$ were then located using Talairach Daemon (Research Imaging Center, University of Texas Health Science Center, San Antonio, TX) and confirmed with the Talairach atlas (Talairach and Tournoux, 1988).

\section{Results}

\section{Behavioral profiles of dyslexic, age-matched, and reading-matched groups}

Demographic, standardized reading test, and vocabulary test [ID-raw, ID-ss(age), WA-ss(age), PC-ss(age), and PPVTss(age)] data for the three groups are presented in Table 1, including the results of one-way ANOVAs and post hoc Tukey tests. ID-raw and ID-ss(age) for each subject are plotted as scattergrams in Figure 1.

One-way ANOVA of PPVT-ss(age) scores revealed no significant effect of group $\left(F_{(2,27)}=1.33 ; p=0.29\right)$. Only the dyslexic group showed a significant difference between ID-ss(age) and PPVT-ss(age) scores $\left(t_{(9)}=2.93 ; p=0.02\right)$; the age-matched $\left(t_{(9)}=1.09 ; p=0.33\right)$ and reading-matched $\left(t_{(9)}=0.24 ; p=\right.$ 0.82 ) groups did not have a discrepancy between reading and vocabulary scores. Thus, the dyslexic group exhibited a disproportionate reading deficit relative to receptive vocabulary and estimated IQ.

Relative to the dyslexic group, there was no significant difference in age for the age-matched group $\left(t_{(18)}=1.66 ; p=0.12\right)$, and no significant difference in reading level for the readingmatched group as determined by ID-raw scores $\left(t_{(18)}=0.08 ; p=\right.$ $0.94)$. Age- and reading-matched groups were well matched by their ID-ss(age) scores $\left(t_{(18)}=0.05 ; p=0.96\right)$, indicating that both groups read similarly well relative to their ages. The agematched group had a higher reading level than the readingmatched group, reflecting two more years of education and experience in reading (ID-raw: $t_{(18)}=6.35 ; p<0.001$ ).

ID scores were used to define groups, but the groups exhibited the same patterns of performance on tests of nonsense word de- 
Table 2. Activations for age-matched, reading-matched, and dyslexic groups, and of all groups combined for rhyme > rest contrast

\begin{tabular}{|c|c|c|c|c|c|c|c|}
\hline \multirow[b]{2}{*}{ Region } & \multirow[b]{2}{*}{ Brodmann area } & \multicolumn{3}{|c|}{ Talairach coordinates } & \multirow[b]{2}{*}{$Z$} & \multirow[b]{2}{*}{$p$} & \multirow[b]{2}{*}{ Volume (ml) } \\
\hline & & $x$ & $y$ & $z$ & & & \\
\hline \multicolumn{8}{|l|}{ Age-matched group } \\
\hline \multicolumn{8}{|l|}{ Frontal lobe } \\
\hline R Middle frontal gyrus & 10 & 46 & 48 & -9 & 3.49 & $<0.001$ & 0.14 \\
\hline \multicolumn{8}{|l|}{ Limbic lobe } \\
\hline L Insula & 13 & -44 & 8 & -2 & 4.01 & $<0.001$ & 1.12 \\
\hline L Cingulate gyrus & 32 & -10 & 19 & 32 & 3.64 & $<0.001$ & 0.24 \\
\hline $\mathrm{R}$ Cingulate gyrus & 32 & 2 & 23 & 39 & 3.93 & $<0.001$ & 2.63 \\
\hline \multicolumn{8}{|l|}{ Temporal lobe } \\
\hline Superior temporal gyrus & 38 & 42 & 19 & -18 & 4.37 & $<0.001$ & 1.26 \\
\hline \multicolumn{8}{|l|}{ Parietal lobe } \\
\hline L Superior parietal lobule & 7 & -26 & -66 & 46 & 3.91 & $<0.001$ & 0.33 \\
\hline R Superior parietal lobule & 7 & 36 & -62 & 49 & 3.57 & $<0.001$ & 0.09 \\
\hline Occipital lobe & & & & & & & \\
\hline L Middle occipital gyrus & 18 & -32 & -95 & 3 & 5.13 & $<0.001$ & 3.64 \\
\hline R Middle occipital gyrus & 18 & 28 & -97 & 9 & 4.6 & $<0.001$ & 2.31 \\
\hline Cerebellum & & & & & & & \\
\hline L Posterior cerebellum & $N / A$ & -8 & -82 & -18 & 3.81 & $<0.001$ & 0.29 \\
\hline R Anterior cerebellum & N/A & 4 & -51 & -4 & 4.85 & $<0.001$ & 1.40 \\
\hline Reading-matched group & & & & & & & \\
\hline Frontal lobe & & & & & & & \\
\hline L Medial frontal gyrus & 6 & -2 & 8 & 51 & 4.58 & $<0.001$ & 2.86 \\
\hline L Inferior frontal gyrus & 45 & -51 & 25 & 4 & 3.33 & $<0.001$ & 0.08 \\
\hline L Precentral gyrus & 6 & -61 & 3 & 24 & 3.78 & $<0.001$ & 0.17 \\
\hline L Precentral gyrus & 6 & -40 & -2 & 37 & 3.51 & $<0.001$ & 0.15 \\
\hline R Medial frontal gyrus & 8 & 8 & 22 & 47 & 3.72 & $<0.001$ & 0.31 \\
\hline R Medial frontal gyrus & 9 & 8 & 29 & 32 & 3.68 & $<0.001$ & 0.19 \\
\hline R Inferior frontal gyrus & 47 & 34 & 23 & -8 & 3.43 & $<0.001$ & 0.21 \\
\hline Limbic lobe & & & & & & & \\
\hline R Insula & 13 & 38 & 18 & 3 & 3.48 & $<0.001$ & 0.30 \\
\hline Temporal lobe & & & & & & & \\
\hline L Superior temporal gyrus & 38 & -44 & 9 & -7 & 4.63 & $<0.001$ & 5.02 \\
\hline L Fusiform gyrus & 37 & -44 & -53 & -12 & 4.48 & $<0.001$ & 0.43 \\
\hline R Fusiform gyrus & 37 & 44 & -53 & -12 & 4.20 & $<0.001$ & 0.24 \\
\hline Occipital lobe & & & & & & & \\
\hline L Inferior occipital gyrus & 18 & -32 & -92 & -6 & 4.33 & $<0.001$ & 5.02 \\
\hline R Middle occipital gyrus & 18 & 26 & -95 & 3 & 4.08 & $<0.001$ & 3.30 \\
\hline Sublobar & & & & & & & \\
\hline L Lentiform nucleus, putamen & & -20 & 2 & -5 & 4.12 & $<0.001$ & 4.03 \\
\hline R Claustrum & $\mathrm{N} / \mathrm{A}$ & 30 & 10 & 1 & 3.84 & $<0.001$ & 0.33 \\
\hline L Thalamus, medial dorsal nucleus & & -10 & -17 & 6 & 3.60 & $<0.001$ & 0.34 \\
\hline Cerebellum & & & & & & & \\
\hline L Cerebellum anterior lobe & $\mathrm{N} / \mathrm{A}$ & -12 & -51 & -18 & 3.46 & $<0.001$ & 0.13 \\
\hline R Cerebellum posterior lobe & $\mathrm{N} / \mathrm{A}$ & 42 & -67 & -17 & 3.97 & $<0.001$ & 0.18 \\
\hline Dyslexic group & & & & & & & \\
\hline Frontal lobe & & & & & & & \\
\hline L Superior frontal gyrus & 6 & -6 & 10 & 53 & 3.79 & $<0.001$ & 0.74 \\
\hline L Inferior frontal gyrus & 44 & -48 & 12 & 12 & 3.84 & $<0.001$ & 0.53 \\
\hline L Precentral gyrus & 4 & -46 & -7 & 48 & 4.57 & $<0.001$ & 0.63 \\
\hline L Precentral gyrus & 6 & -53 & 1 & 13 & 3.86 & $<0.001$ & 0.22 \\
\hline L Precentral gyrus & 6 & -59 & -4 & 30 & 3.31 & $<0.001$ & 0.13 \\
\hline Occipital lobe & & & & & & & \\
\hline L Middle occipital gyrus & 18 & -28 & -95 & 7 & 4.54 & $<0.001$ & 3.46 \\
\hline$R$ Cuneus & 18 & 24 & -97 & -2 & 4.97 & $<0.001$ & 2.45 \\
\hline All groups combined & & & & & & & \\
\hline Frontal, limbic lobes, sublobar & & & & & & & \\
\hline L Superior, medial frontal, cingulate gyri & $6,8,32$ & -4 & 8 & 53 & 6.09 & $<0.001$ & 16.55 \\
\hline L Inferior frontal, precentral gyrus, insula & $47,13,6$ & -40 & 17 & -3 & 5.52 & $<0.001$ & 30.50 \\
\hline R Inferior frontal gyrus, insula, lentiform nucleus & 13,47 & 40 & 13 & -4 & 4.86 & $<0.001$ & 8.18 \\
\hline R Inferior frontal, precentral gyrus & 6,9 & 63 & -1 & 18 & 3.51 & $<0.001$ & 0.83 \\
\hline Temporal lobe & & & & & & & \\
\hline L Hippocampus, lentiform nucleus & & -32 & -20 & -7 & 3.58 & $<0.001$ & 0.26 \\
\hline Occipital lobe & & & & & & & \\
\hline L Middle, inferior occipital gyri & 18 & -28 & -95 & 5 & 7.11 & $<0.001$ & 11.12 \\
\hline R Middle, inferior occipital, fusiform gyri & 18 & 28 & -95 & 3 & 7.05 & $<0.001$ & 8.32 \\
\hline Sublobar & & & & & & & \\
\hline R Thalamus & & 14 & -17 & 6 & 3.6 & $<0.001$ & 0.28 \\
\hline
\end{tabular}

$p=0.001, \mathrm{ET}, 10$; uncorrected. N/A, Not applicable. 
coding (WA scores) and comprehension (PC scores) (Table 1). There were no significant differences between the two control groups for age-adjusted scores [i.e., both control groups were reading at similar and normal levels relative to their ages; WAss(age): $t_{(18)}=0.38, p=0.71$; PC-ss(age): $t_{(18)}=0.35, p=0.73$ ] Similar to the ID-ss(age) results, the dyslexic group had significantly lower scores compared with the age-matched [WAss(age): $t_{(18)}=4.11, p=0.001 ;$ PC-ss(age): $\mathrm{t}_{(18)}=4.73, p<$ 0.001 ], and the reading-matched [WA-ss(age): $t_{(18)}=4.46, p<$ 0.001 ; PC-ss(age): $\left.t_{(18)}=5.15, p<0.001\right]$ groups. Dyslexic and reading-matched groups, however, showed no significant difference in their raw reading scores (WA-raw: $t_{(18)}=0.77, p=0.45$; PC-raw: $\left.t_{(18)}=0.51, p=0.62\right)$, as was the case for ID-raw.

\section{Scanner task performance}

A one-way ANOVA revealed a significant difference on scanner-task accuracy and RT for correct trials ( $p$ values $<0.05$ ), but not for RT for incorrect trials $(p=0.67)$ among the three groups (Table 1). Post hoc analysis for accuracy and correct RT showed that this effect was caused by significantly worse accuracy and a trend toward longer RTs for correct trials in the dyslexic group than the age-matched group (post hoc Tukey, accuracy, $p=0.01$; correct RT, $p=0.06$ ). There was no significant difference between dyslexic and reading-matched groups ( post hoc Tukey, accuracy, $p=0.28$; correct RT, $p=$ $1.00)$.

\section{Activation patterns in normal-reading and} dyslexic children

Whole-brain analyses for each of the agematched, reading-matched, and dyslexic groups (Table 2), and for all subjects combined (Fig. 2, Table 2) revealed significant activation in multiple regions known to be associated with phonological processing.

\section{Comparisons between normal-reading and} dyslexic children

\section{Comparison between age-matched and}

dyslexic children

The age-matched group had activation significantly greater than the dyslexic group in six brain regions: two regions in left parietotemporal cortex, one region in right parietotemporal cortex, two frontal regions, and one region in right occipitotemporal cortex (Fig. 3, Table 3). The dyslexic group did not show any regions of activation significantly greater than the agematched group (Table 3). Regions identified using a one-way ANOVA whole-brain analyses $F$ test (age-matched vs readingmatched vs dyslexic groups) also showed similar regions (supplemental Fig. 1 and Table 2, available at www.jneurosci.org as supplemental material).

Comparisons between age-matched, reading-matched, and dyslexic children

Having identified six ROIs showing greater activation in agematched than dyslexic children, the critical analysis compared activation between reading-matched and dyslexic children in

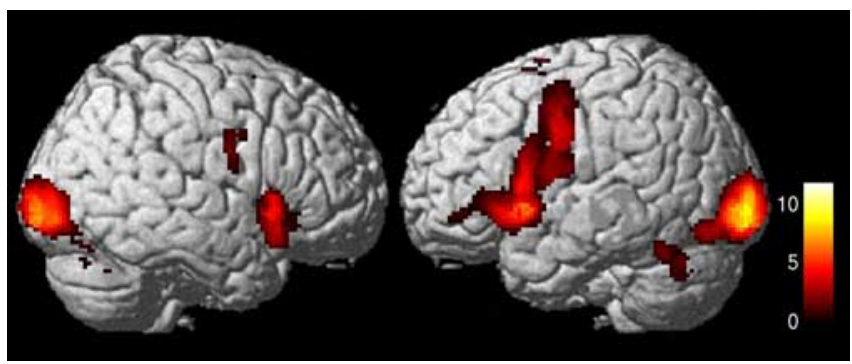

Figure 2. Whole brain activation for the contrast rhyme $>$ rest. The main effect of condition for the three groups is examined and the activation map is rendered on a 3D brain ( $p=0.001 ; \mathrm{ET}, 10)$.

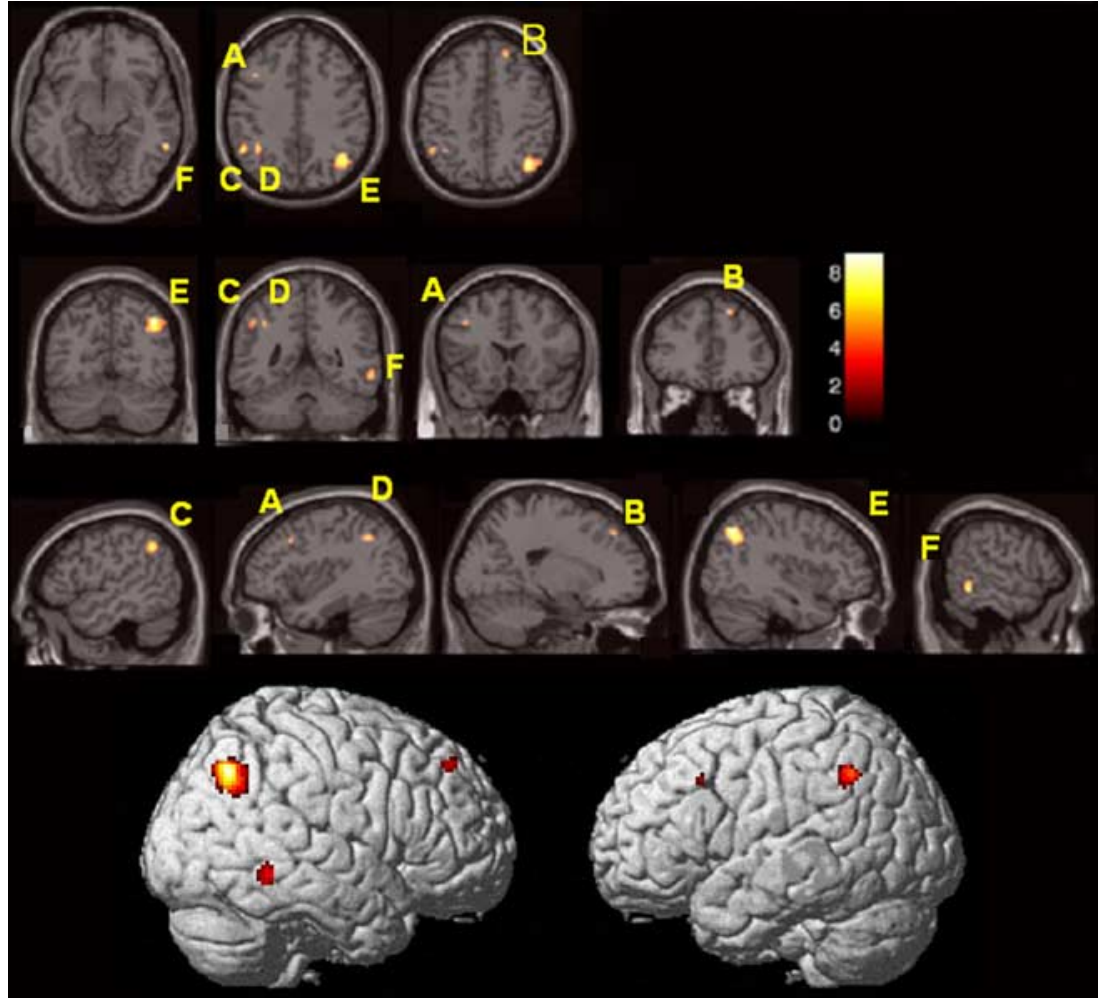

Figure 3. Comparison between age-matched and dyslexic groups. Results from the two-sample $t$ test of the contrast images rhyme $>$ rest with greater activation in the age-matched compared with dyslexic groups overlaid on a normalized single subject axial, coronal, and sagittal views of the brain and on a 3D rendered brain ( $p=0.001$; ET, 10). Top, Axial view of Talairach coordinate (TC), z: -6, 38, 44; second row, coronal view TC $y$ : $-62,-49,19$, 41; third row, sagittal view TC $x$ $-50,-38,20,59,40$; bottom, 3D rendered brain. Brain regions: $A$, left middle frontal gyrus; $B$, right superior frontal gyrus; C, left inferior parietal lobule; $D$, left inferior parietal lobule; $E$, right inferior parietal lobule; $F$, right posterior middle temporal gyrus. Activation difference coordinates are shown in Table 3. those same six regions. One-way ANOVAs for each of the six ROIs were performed in the dyslexic, age-matched, and readingmatched groups (Fig. 4A). All six regions showed a significant effect of group ( $p$ values $<0.05$ ) (Table 4$)$. Significant differences between age-matched and dyslexic groups were expected given how these regions were selected (i.e., from the results agematched $>$ dyslexic groups; $p$ values $<0.05$ ) (Table 4). Critically, the reading-matched group exhibited greater activation than the dyslexic group in all six regions ( $p$ values $<0.05$; Table 4 ). Additionally, the age-matched group showed significantly more activation than the reading-matched group in both left and right parietotemporal cortices $(p$ values $<0.05)$ (Table 4$)$.

To examine whether activation differences were arising primarily from the rhyme or rest conditions, we examined $\beta$ esti- 
Table 3. Activation differences between age-matched and dyslexic groups for rhyme $>$ rest contrast

\begin{tabular}{|c|c|c|c|c|c|c|c|}
\hline \multirow[b]{2}{*}{ Region } & \multirow[b]{2}{*}{ Brodmann area } & \multicolumn{3}{|c|}{ Talairach coordinates } & \multirow[b]{2}{*}{$Z$} & \multirow[b]{2}{*}{$p$} & \multirow[b]{2}{*}{ Volume (ml) } \\
\hline & & $x$ & $y$ & $z$ & & & \\
\hline \multicolumn{8}{|l|}{$\begin{array}{l}\text { Age-matched }>\text { dyslexic } \\
\text { Frontal lobe }\end{array}$} \\
\hline $\begin{array}{l}\text { L Middle frontal gyrus } \\
\text { R Superior frontal gyrus }\end{array}$ & $\begin{array}{l}8 \\
9\end{array}$ & $\begin{array}{r}-38 \\
20\end{array}$ & $\begin{array}{l}19 \\
41\end{array}$ & $\begin{array}{l}34 \\
38\end{array}$ & $\begin{array}{l}3.38 \\
3.63\end{array}$ & $\begin{array}{l}<0.001 \\
<0.001\end{array}$ & $\begin{array}{l}0.08 \\
0.22\end{array}$ \\
\hline Parietal lobe & & & & & & & \\
\hline L Inferior parietal lobule 1 & 40 & -50 & -49 & 39 & 4.23 & $<0.001$ & 0.60 \\
\hline L Inferior parietal lobule 2 & 40 & -36 & -49 & 37 & 4.11 & $<0.001$ & 0.41 \\
\hline $\begin{array}{l}\text { R Inferior parietal lobule } \\
\text { Temporal lobe }\end{array}$ & 39 & 40 & -62 & 44 & 5.48 & $<0.001$ & 2.68 \\
\hline $\begin{array}{l}\text { R Middle temporal gyrus } \\
\text { Dyslexic }>\text { age-matched } \\
\text { n/a }\end{array}$ & 37 & 59 & -47 & -6 & 3.92 & $<0.001$ & 0.42 \\
\hline
\end{tabular}

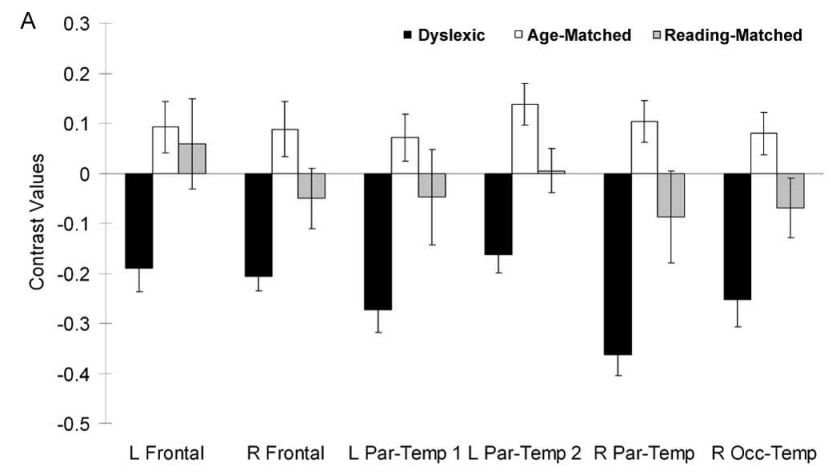
Regions

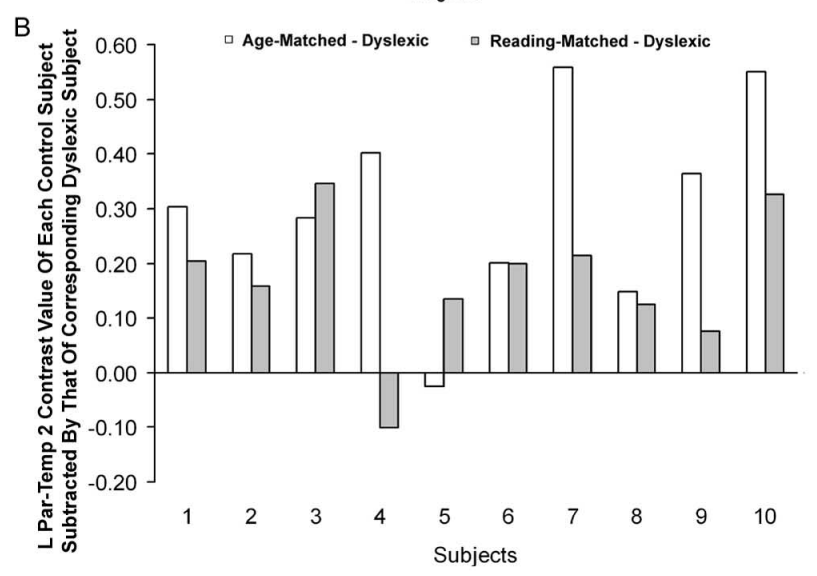

Figure 4. Brain activation patterns of the contrast rhyme $>$ rest comparing three groups. $\boldsymbol{A}$, Brain activation in the six functionally defined ROls in the three groups. ROls were selected based on the comparison age-matched $>$ dyslexic groups in Figure 3. Bar graphs represent the mean contrast values of the six regions for dyslexic (black bars), age-matched (white bars), and reading-matched groups (gray bars). Error bars represent SEM. Brain regions: $L$ Frontal, left middle frontal gyrus (corresponding to region $A$ in Fig. 3); R Frontal, right superior frontal gyrus (corresponding to region B in Fig. 3); L Par-temp 1, left inferior parietal lobule (corresponding to region ( in Fig. 3); L Par-temp 2, left inferior parietal lobule (corresponding to region D in Fig. 3); R Par-temp, right left inferior parietal lobule (corresponding to region E in Fig. 3); R Occ-temp, right posterior middle temporal gyrus (corresponding to region F in Fig. 3). All six one-way ANOVAs were significant ( $p$ values $<0.05$ ) (see also Table 4). Age-matched versus dyslexic, All $p$ values $<$ 0.05 ; reading-matched versus dyslexic, all $p$ values $<0.05$; age-matched versus readingmatched groups, L Par-temp 2, R Par-temp, $p$ values $<0.05$; R Frontal, R 0cc-temp, $p$ values $<0.10$. B Brain activation comparing each dyslexic subject and their corresponding control. Each dyslexic child's contrast value of the L Par-temp 2 was subtracted from the corresponding control child (age-matched, white; reading-matched, gray) and is plotted so that zero equals the activation value of each of the 10 dyslexic subjects. Positive values indicate that the individually matched control child had greater activation than their corresponding dyslexic child. Nine of 10 children for each control group showed greater activation than their corresponding dyslexic child. mates separately for the two conditions for each of the six regions. For $\beta$ estimates of the resting baseline condition, there was a main effect of group only in the right parietotemporal region (R Par-temp; $F_{(2,27)}=$ 3.92; $p=0.02$ ), which was driven by the significantly greater $\beta$ values in the reading-matched $\left(t_{(18)}=2.5 ; p<0.02\right)$ and age-matched $\left(t_{(18)}=2.4 ; p=0.01\right)$ groups compared with the dyslexic group. The rhyme condition $\beta$ estimates showed significant main effects (or a trend) of group in five of the six regions [right superior frontal gyrus (R Frontal): $F_{(2,27)}=3.44$, $p=0.02$; left inferior parietal lobule $1(\mathrm{~L}$ Par-temp 1): $F_{(2,27)}=3.00, p=0.03 ; \mathrm{L}$ Par-temp 2: $F_{(2,27)}=3.87$, $p=0.02$; R Par-temp: $F_{(2,27)}=13.85, p<0.001$; right posterior middle temporal gyrus (R Occ-temp): $\left.F_{(2,27)}=2.21, p=0.07\right]$. These were driven by the significantly greater (or trends for) $\beta$ values in the reading-matched and/or age-matched compared with the dyslexic groups (reading-matched $>$ dyslexic: L Partemp 2, $t_{(18)}=1.92, p=0.04$; R Par-temp, $t_{(18)}=3.77, p<0.001$; R Occ-temp, $t_{(18)}=1.86, p=0.04$; age-matched $>$ dyslexic: $\mathrm{R}$ Frontal, $t_{(18)}=2.67, p=0.01$; L Par-temp 1, $t_{(18)}=2.25, p=$ 0.02; L Par-temp 2, $t_{(18)}=2.69, p=0.01$; R Par-temp, $t_{(18)}=$ 5.13, $p<0.001$; R Occ-temp, $\left.t_{(18)}=1.56, p=0.07\right)$. Other regions not listed here did not show a main effect of group for the resting baseline or rhyme condition $\beta$ estimates (all $p$ values $>$ 0.05). These results show that the group differences in the rhyme $>$ rest contrast were driven more by dyslexic differences in the rhyme condition than the resting baseline. This was especially true in the left and right parietotemporal regions (L Par-temp 2 and R Par-temp) in which the dyslexic group showed significantly less activation in the rhyme condition than both the agematched and the reading-matched groups.

These group differences were evident in each of the six ROIs when each dyslexic participant's activation was compared with his or her individually selected age-matched or reading-matched control. For example, in the left parietotemporal region (L Partemp 2), 9 of 10 dyslexic children showed lower activation than their age-matched or reading-matched counterparts (Fig. 5). Across all six ROIs, depending on the ROI, 9 to 10 of the 10 dyslexic children showed less activation than their corresponding age-matched child, and 7 to 9 of the 10 dyslexic children showed less activation than their corresponding reading-matched child, despite their matched reading proficiency (ID-raw score). Binomial tests showed that for the dyslexic and age-matched counterparts, all six regions showed a significant effect (i.e., significantly greater proportion than 0.5 with a two-tailed test) (L Frontal, $\mathrm{R}$ Frontal, L Par-temp 1, L Par-temp 2, $p=0.02$; R Par-temp, R Occ-temp, $p=0.002$ ). For the dyslexic and reading-matched counterparts, the left and right parietotemporal regions showed significant effects (L Par-temp 2, R Par-temp, $p=0.02$; L Frontal, $p=0.34$; R Frontal, L Par-temp 1, R Occ-temp, $p=0.11)$. Hence, by a binomial test, the dyslexic group showed a reliable decrease in activation in left and right parietotemporal regions relative to both age-matched and reading-matched groups.

An unexpected aspect of the findings was the relation between activation for the rhyme condition and the resting baseline. The dyslexic group showed reliably greater activation for the resting baseline than the rhyming task in all six regions $\left(t_{(9)}=\right.$ $4.09 \sim 8.69 ; p=0.003 \sim<0.001)$. The reading-matched group showed similar activation in the rhyme task and resting baseline, 
and only the age-matched group showed greater activation for the rhyming task, but this reached significance only in the left ( $\mathrm{L}$ Par-temp 2, $\left.t_{(9)}=3.31 ; p=0.009\right)$ and right parietotemporal regions $\left(t_{(9)}=2.52\right.$; $p=0.003)$. Similar results were obtained when the same analyses were performed without global scaling.

\section{Correlation between brain activation and reading ability}

To examine whether activation in the left parietotemporal cortex correlated with reading ability, correlation analyses were performed between IDss(age) scores and the magnitude of activation in the left parietotemporal cortex for all 30 participants (L Par-temp 2) (Fig. 5). There was a positive and significant correlation $(r=0.50 ; p=$ 0.002). The other left parietotemporal region (L Par-temp 1) showed similar correlation $(r=0.48)$. Activation in the left parietotemporal region correlated also with other reading scores [WA-ss(age), $r=0.55, p<0.001$; PC-ss(age), $r=0.39, p=$ 0.018 ] and scanner task performance $(r=0.44 ; p=0.02)$. These significant correlations mainly reflected group difference as each group on its own did not show even a trend toward a significant correlation ( $p$ values $>0.42$ ). The absence of correlations within groups may reflect limited power, and also a restriction of range within groups resulting from the initial assignment of subjects into matched groups.

\section{Relation of scanner task performance to brain activation}

There was a large and significant difference in scanner task performance between dyslexic and age-matched groups (19.5\%). The difference between dyslexic and reading-matched groups was smaller $(9.5 \%)$ and nonsignificant $(p>0.10)$, but that difference could reach significance with a larger sample. To investigate whether our results reflect differences in scanner task performance (with reading-level also controlled) between groups, we compared 14 subjects (seven per group) from the dyslexic and reading-matched groups strictly matched for scanner task performance (dyslexic: mean, 79.3; SD, 10.6; reading-matched: mean, 79.3; SD, 7.3; $t$ test: $t_{(12)}=0.00, p=1.00$ ), and still found significantly reduced activation for the dyslexic compared with the reading-matched group in the left parietotemporal (L Par-temp $\left.1, t_{(12)}=2.30, p=0.04\right)$, right parietotemporal $\left(t_{(12)}=3.70 ; p=\right.$ $0.003)$ and right frontal regions $\left(t_{(12)}=2.35 ; p=0.04\right)$, with a trend for significance in the second left parietotemporal region ( $\mathrm{L}$ Par-temp 2, $\left.t_{(12)}=2.1, p=0.06\right)$. The dyslexic and readingmatched subsamples were still well matched for their reading level [ID-raw, $t_{(12)}=0.52, p=0.61$; WA-raw, $t_{(12)}=0.40, p=$ 0.70 ; PC-raw(age), $t_{(12)}=0.56, p=0.59$ ]. These results indicate that the results reported here are not simply consequences of differences in scanner task performance (supplemental analyses, available at www.jneurosci.org as supplemental material, report other analyses supporting this finding), because the key differences in activation remained when the dyslexic and readingmatched groups were equated for both scanner task performance and reading level (with some differences no longer statistically reliable because of decreased power in the smaller sample).

\section{Discussion}

The dyslexic children in the present study exhibited impaired phonological performance on the in-scanner rhyme task and on standardized tests of phonological processing, single-word reading, and passage comprehension. Consistent with previous func-

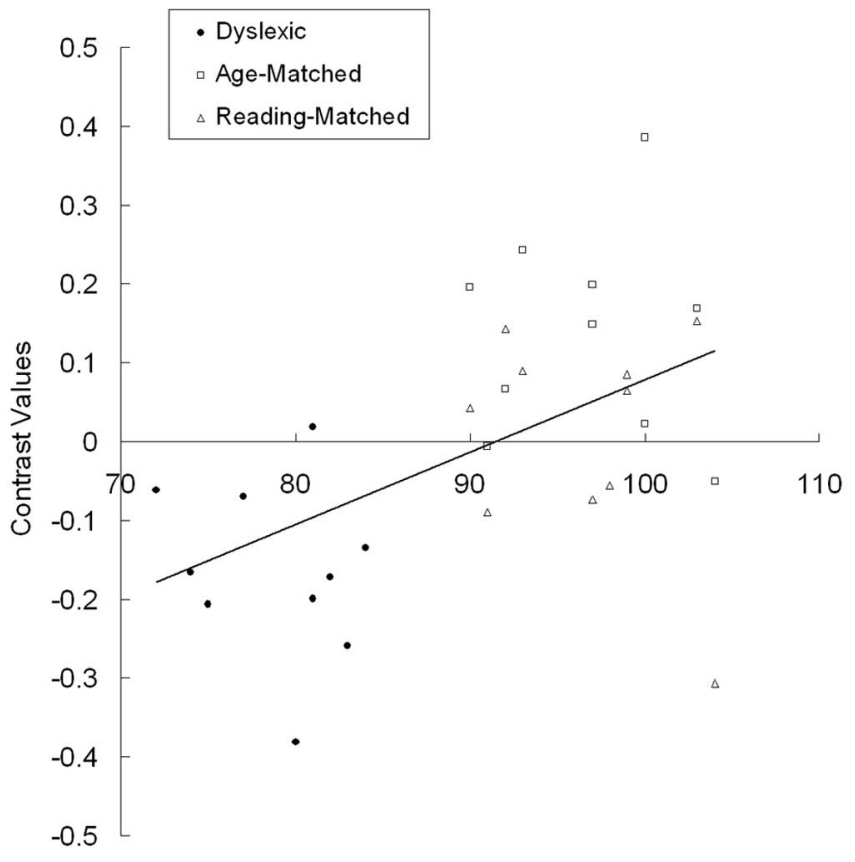

WordID-ss[age]

Figure 5. Scatter plot showing the correlation between brain activation and reading scores and the correlation between word ID-ss(age) scores and the L Par-temp 2 contrast values in the dyslexic (filled circles), age-matched (open squares), and reading-matched groups (open triangles, $n=30) . r=0.50 ; p=0.0023$.

tional neuroimaging studies of phonological processing in dyslexia, we found reduced left parietotemporal activation in dyslexic children compared with age-matched controls. This same region was also significantly less activated in dyslexic children than a group of normal readers with equal reading ability (reading-matched controls) or reading-matched controls equal also in scanner performance (scanner-matched controls). The same pattern of reduced activation in dyslexia relative to readingmatched controls was found in five other brain regions. Our results thus indicate that the reduced activation in the left parietotemporal region and other brain regions observed here is characteristic of dyslexia, and not a consequence of either low reading proficiency or impaired in-scanner performance.

Multiple functional neuroimaging studies of phonological processing in dyslexia have reported reduced activation in left parietotemporal cortex, both in adults (Rumsey et al., 1992, 1999; Paulesu et al., 1996; Horwitz et al., 1998; Shaywitz et al., 1998; Brunswick et al., 1999; Ruff et al., 2002), and in children (Shaywitz et al., 2002; Simos et al., 2000a,b). The importance of the phonological processes supported by this region for reading is indicated not only by its consistent hypoactivation in dyslexia, but also by the growth of activations in this region associated with 
effective remediation in adults (Eden et al., 2004) and children (Simos et al., 2002; Temple et al., 2003).

The specific location of left parietotemporal hypoactivation in dyslexia has varied across studies, perhaps reflecting variation in tasks, analyses, or participants. The importance of the left parietotemporal region showing hypoactivation in the dyslexic group in the present study is supported by significant correlations between the magnitudes of activation in this region and standardized tests of word reading, pseudoword decoding, passage comprehension, and in-scanner performance, as well as a significant growth of activation from third to fifth grade in typically developing readers.

Previous neuroimaging studies, however, contrasted dyslexic groups with control groups exhibiting superior reading performance, and often, superior in-scanner performance. The difference in activation patterns between age-matched and dyslexic groups could have been attributable to many factors, such as differences in reading skills, in-scanner performance, effort, attention, error monitoring, and motivation. These factors were not relevant in the comparison between the dyslexic fifth graders and reading-matched or scanner-task-matched third graders studied here. The dyslexic group showed reduced activation relative to reading-matched third graders in all six regions for which they showed reduced activation relative to age-matched fifth graders. Also, a dyslexic subgroup matched to third graders for both in-scanner performance and reading level exhibited significantly reduced activations in three regions, including the left parietotemporal cortex. Further, when dyslexic children were individually compared with their corresponding controls for left parietotemporal activation, 9 of 10 dyslexic children had lower activation than their corresponding age-matched control child, and 9 of 10 dyslexic children had lower activation than their corresponding reading-matched control child. Thus, these findings show that the reduced parietotemporal activations in these dyslexic children cannot be accounted for by reading ability or in-scanner performance. Rather, the hypoactivation reflects an atypical brain substrate for reading.

Normal developmental differences in the neural correlates of phonological processing could be examined by comparing the normal reading third and fifth graders. The fifth graders scored better on all tests of reading, as would be expected after two more years of maturation, reading instruction, and reading practice. Activation increased in all six regions from the third to the fifth graders, significantly in both left and right parietotemporal regions, and trending toward significance in right occipitotemporal and frontal regions. Activation in two of the parietotemporal regions did not correlate with scanner performance within the 20 control children, so these activation differences are associated with developmental differences in reading and language abilities rather than in-scanner performance.

Reading-related developmental changes in brain activation have been examined in larger samples with other tasks, and compared either children of ages 7-10 with adults (Schlaggar et al., 2002) or a more continuous sampling from ages 6-22, with most subjects clustering 12 years or below and 20 years or above (Turkeltaub et al., 2003). These studies reported growths of activation, when controlling for performance, in left frontal and other left hemisphere areas. Our findings may differ because of the selection of a phonological task, a resting baseline, and/or a far smaller developmental period of two years versus the decade or more examined in the previous imaging studies. The present findings indicate that fMRI can be sensitive to the maturation of brain functions associated with a mere two years of cross-sectional normal reading development.

Clearly, the dyslexic group was not simply developmentally delayed in the sense of having the same brain-behavior relations as the reading-matched third graders. The dyslexic pattern of activations could possibly resemble an even younger and worse reading group, such as normal-reading first graders. Our findings, however, indicate that dyslexic children are not on the normal developmental trajectory of brain-behavior relations in the neural systems that support reading. The present data are consistent with the widespread observation that although many dyslexic individuals compensate to some extent for their dyslexia by adulthood, they do not catch up with typical individuals in terms of comfort and efficiency in reading (i.e., dyslexia is not a simple delay in reading proficiency). The hypoactivation of the left and right parietotemporal regions in dyslexia may mark an important brain dysfunction in dyslexia, because these are the very regions that appear to be most associated with normal gains in reading from third to fifth grades.

An unexpected finding was that the dyslexic deficit in activation reflected a large deactivation for the rhyme task compared with the rest condition in dyslexic children (here "deactivation" is defined as greater activation for a lower-order baseline task than the more demanding higher-order activation task). Analyses of each condition indicated that activation in the rhyme condition was a larger contributor to group differences than activation in the rest condition, but the rest condition also contributed to the group differences. Many studies have reported activation differences between normal and poor readers, but did not tease apart whether this difference was caused by more activation for the higher-order task in the normal readers or by more activation for the lower-order baseline task in the poor readers, or both. For example, in a study by Shaywitz et al. (2002), greater activation in the left angular and temporal regions in normal than dyslexic children may be caused by deactivation by the dyslexic group in the rhyme and category conditions, as no significant activation was observed in these regions in the normal readers. Ruff et al. (2002) also showed deactivation in the left parietotemporal region during implicit categorical perception of phonemes in dyslexic adults. Thus, a reversal in activation across conditions between normal and dyslexic readers may often occur in the left parietotemporal cortex.

Five other regions exhibited reduced activation in dyslexic children relative to both age-matched and reading-matched children: a second left parietotemporal region, right parietotemporal cortex, right occipitotemporal cortex, and bilateral frontal cortices. Right parietotemporal hypoactivation has been reported in adults with dyslexia (Rumsey et al., 1997) and this area shows increased activation after remediation (Eden et al., 2004). The right occipitotemporal region has been reported to be hypoactive in dyslexia in some studies (Rumsey et al., 1997; Shaywitz et al., 2002), and this region has shown increased activation with successful remediation in dyslexic children (Temple et al., 2003). Finally, the left frontal region is of interest because this was the only region other than the left parietotemporal region (L Pattemp 1), that showed no significant difference between age- and reading-matched controls (other regions showed significant or trends toward significant differences). Thus, the dyslexic group exhibited reduced activations relative to reading-matched children both in regions that showed developmental trends, and also in regions that did not show developmental trends. Reduced activation in dyslexia (Aylward et al., 2003; Temple et al., 2003) and an increase in activation after successful remediation (Temple et 
al., 2000, 2003; Eden et al., 2004) have been reported in the left frontal region. Future investigation is warranted to further elucidate the different roles of these regions.

The present findings indicate that reduced activation in dyslexia during phonological processing cannot be explained by either current reading ability or in-scanner performance. A limitation of this study is that we did not include another group with poor reading attributable to another etiology, such as autism. Inclusion of another atypical reading group could shed light on whether the observed patterns of atypical activation are specific to dyslexia per se, or whether they also extend to other developmental reading disorders. Our findings do indicate, however, that hypoactivation during phonological processing marks brain regions that may play a critical role in the neural basis of dyslexia.

\section{References}

Aylward EH, Richards TL, Berninger VW, Nagy WE, Field KM, Grimme AC, Richards AL, Thomson JB, Cramer SC (2003) Instructional treatment associated with changes in brain activation in children with dyslexia. Neurology 61:212-219.

Bradley L, Bryant PE (1978) Difficulties in auditory organisation as a possible cause of reading backwardness. Nature 271:746-747.

Bradley L, Bryant PE (1983) Categorizing sounds and learning to read: a causal connection. Nature 301:419-421.

Brunswick N, McCrory E, Price CJ, Frith CD, Frith U (1999) Explicit and implicit processing of words and pseudowords by adult developmental dyslexics: a search for Wernicke's Wortschatz? Brain 122:1901-1917.

Burgund ED, Kang HC, Kelly JE, Buckner RL, Snyder AZ, Petersen SE, Schlaggar BL (2002) The feasibility of a common stereotactic space for children and adults in fMRI studies of development. NeuroImage 17:184-200.

Castles A, Coltheart M (2004) Is there a causal link from phonological awareness to success in learning to read? Cognition 91:77-111.

Dunn LM, Dunn LM (1997) Examiner's manual for the Peabody picture vocabulary test, Ed 3. Circle Pines, MN: American Guidance Service.

Eden GF, Jones KM, Cappell K, Gareau L, Wood FB, Zeffiro TA, Dietz NA, Agnew JA, Flowers DL (2004) Neural changes following remediation in adult developmental dyslexia. Neuron 44:411-422.

Elbro C, Bosrstrom I, Petersen DK (1998) Predicting phonological awareness from kindergarten: the importance of distinctness of phonological representations of lexical items. Read Res Q 33:36-60.

Friston KJ, Holmes AP, Price CJ, Buchel C, Worsley KJ (1999) Multisubject fMRI studies and conjunction analyses. NeuroImage 10:385-396.

Horwitz B, Rumsey JM, Donohue BC (1998) Functional connectivity of the angular gyrus in normal reading and dyslexia. Proc Natl Acad Sci USA 95:8939-8944.

Jenkinson M, Bannister P, Brady M, Smith S (2002) Improved optimization for the robust and accurate linear registration and motion correction of brain images. NeuroImage 17:825-841.

Kang HC, Burgund ED, Lugar HM, Petersen SE, Schlaggar BL (2003) Comparison of functional activation foci in children and adults using a common stereotactic space. NeuroImage 19:16-28.

Lundberg I, Olofsson A, Wall S (1980) Reading and spelling skills in the first school years predicted from phonemic awareness skills in kindergarten. Scand J Psychol 21:159-173.

MacDonald GW, Cornwall A (1995) The relationship between phonological awareness and reading and spelling achievement eleven years later. J Learn Disabil 28:523-527.

Paulesu E, Frith U, Snowling M, Gallagher A, Morton J, Frackowiak RS, Frith CD (1996) Is developmental dyslexia a disconnection syndrome? Evidence from PET scanning. Brain 119:143-157.

Ruff S, Cardebat D, Marie N, Demonet JF (2002) Enhanced response of the left frontal cortex to slowed down speech in dyslexia: an fMRI study. NeuroReport 13:1285-1289.

Rumsey JM, Andreason P, Zametkin AJ, Aquino T, King AC, Hamburger SD, Pikus A, Rapoport JL, Cohen RM (1992) Failure to activate the left temporoparietal cortex in dyslexia. An oxygen 15 positron emission tomographic study. Arch Neurol 49:527-534.
Rumsey JM, Nace K, Donohue B, Wise D, Maisog JM, Andreason P (1997) A positron emission tomographic study of impaired word recognition and phonological processing in dyslexic men. Arch Neurol 54:562-573.

Rumsey JM, Horwitz B, Donohue BC, Nace KL, Maisog JM, Andreason P (1999) A functional lesion in developmental dyslexia: left angular gyral blood flow predicts severity. Brain Lang 70:187-204.

Schlaggar BL, Brown TT, Lugar HM, Visscher KM, Miezin FM, Petersen SE (2002) Functional neuroanatomical differences between adults and schoolage children in the processing of single words. Science 296:1476-1479.

Shaywitz BA, Shaywitz SE, Pugh KR, Mencl WE, Fulbright RK, Skudlarski P, Constable RT, Marchione KE, Fletcher JM, Lyon GR, Gore JC (2002) Disruption of posterior brain systems for reading in children with developmental dyslexia. Biol Psychiatry 52:101-110.

Shaywitz BA, Shaywitz SE, Blachman BA, Pugh KR, Fulbright RK, Skudlarski P, Mencl WE, Constable RT, Holahan JM, Marchione KE, Fletcher JM, Lyon GR, Gore JC (2004) Development of left occipitotemporal systems for skilled reading in children after a phonologically-based intervention. Biol Psychiatry 55:926-933.

Shaywitz SE (1998) Dyslexia. N Engl J Med 338:307-312.

Shaywitz SE, Shaywitz BA (2003) Dyslexia (specific reading disability). Pediatr Rev 24:147-153.

Shaywitz SE, Shaywitz BA, Pugh KR, Fulbright RK, Constable RT, Mencl WE, Shankweiler DP, Liberman AM, Skudlarski P, Fletcher JM, Katz L, Marchione KE, Lacadie C, Gatenby C, Gore JC (1998) Functional disruption in the organization of the brain for reading in dyslexia. Proc Natl Acad Sci USA 95:2636-2641.

Shaywitz SE, Fletcher JM, Holahan JM, Shneider AE, Marchione KE, Stuebing KK, Francis DJ, Pugh KR, Shaywitz BA (1999) Persistence of dyslexia: the Connecticut Longitudinal Study at adolescence. Pediatrics 104:1351-1359.

Simos PG, Breier JI, Fletcher JM, Bergman E, Papanicolaou AC (2000a) Cerebral mechanisms involved in word reading in dyslexic children: a magnetic source imaging approach. Cereb Cortex 10:809-816.

Simos PG, Breier JI, Fletcher JM, Foorman BR, Bergman E, Fishbeck K, Papanicolaou AC (2000b) Brain activation profiles in dyslexic children during non-word reading: a magnetic source imaging study. Neurosci Lett 290:61-65.

Simos PG, Fletcher JM, Bergman E, Breier JI, Foorman BR, Castillo EM, Davis RN, Fitzgerald M, Papanicolaou AC (2002) Dyslexia-specific brain activation profile becomes normal following successful remedial training. Neurology 58:1203-1213.

Sprenger-Charolles L, Cole P, Lacert P, Serniclaes W (2000) On subtypes of developmental dyslexia: evidence from processing time and accuracy scores. Can J Exp Psychol 54:87-104.

Stanovich KE (1999) The sociopsychometrics of learning disabilities. J Learn Disabil 32:350-361.

Stanovich KE, Siegel LS, Gottardo A (1997) Converging evidence for phonological and surface subtypes of reading disability. J Educ Psychol 89:114-127.

Talairach J, Tournoux P (1988) Co-planar stereotaxic atlas of the human brain. New York, NY: Thieme.

Temple E (2002) Brain mechanisms in normal and dyslexic readers. Curr Opin Neurobiol 12:178-183.

Temple E, Poldrack RA, Protopapas A, Nagarajan S, Salz T, Tallal P, Merzenich MM, Gabrieli JD (2000) Disruption of the neural response to rapid acoustic stimuli in dyslexia: evidence from functional MRI. Proc Natl Acad Sci USA 97:13907-13912.

Temple E, Poldrack RA, Salidis J, Deutsch GK, Tallal P, Merzenich MM, Gabrieli JD (2001) Disrupted neural responses to phonological and orthographic processing in dyslexic children: an fMRI study. NeuroReport 12:299-307.

Temple E, Deutsch GK, Poldrack RA, Miller SL, Tallal P, Merzenich MM, Gabrieli JD (2003) Neural deficits in children with dyslexia ameliorated by behavioral remediation: evidence from functional MRI. Proc Natl Acad Sci USA 100:2860-2865.

Turkeltaub PE, Gareau L, Flowers DL, Zeffiro TA, Eden GF (2003) Development of neural mechanisms for reading. Nat Neurosci 6:767-773.

Zeno SM, Ivens SH, Millard RT, Duvvuri R (1995) The educator's word frequency guide. New York, NY: Touchstone Applied Science. 